\title{
O PAPEL DA ENFERMEIRA NA REABILITAÇÃO DO IDOSO
}

Maria José D'Elboux Diogo*

DIOGO, M.J.D’E. O papel da enfermeira na reabilitação do idoso. Rev.latino-am.enfermagem, Ribeirão Preto, v. 8, n. 1, p. $75-81$, janeiro 2000 .

Com o crescente aumento da população idosa em nosso país, torna-se cada vez mais freqüente a presença de idosos nos serviços de saúde e em especial nos de reabilitação. Trazemos neste texto descritivo os principais aspectos envolvidos na reabilitação do idoso: as peculiaridades referentes à reabilitação nesta faixa etária, a avaliação funcional como um importante indicativo da qualidade de vida, as fases da reabilitação do idoso e a assistência de enfermagem sistematizada, por meio da qual a enfermeira, inserida na equipe multidisciplinar atua com o idoso e sua família, de maneira individualizada.

UNITERMOS: reabilitação geriátrica, enfermagem geriátrica e gerontológica

\section{A REABILITAÇÃO DO IDOSO}

O crescente aumento da população idosa em nosso meio, tem sido constatado por estudos demográficos, os quais demonstram que no ano de 2025 o Brasil ocupará o $6^{\circ}$ lugar no mundo, com aproximadamente 34 milhões de idosos (KALACHE et al., 1987). Esta "explosão do número de idosos", já podemos perceber ao nosso redor, em diferentes locais e nos serviços de saúde, especificamente no setor de reabilitação.

Em nosso país, a reabilitação do idoso ainda é um assunto pouco abordado na literatura, o que dificulta o desenvolvimento de trabalhos nesta área. Assim, o objetivo deste texto descritivo é apontar os principais aspectos envolvidos nesta questão, com base na literatura sobre o tema e na nossa experiência profissional, com a finalidade de oferecer subsídios para reflexão sobre a atuação da enfermeira na reabilitação do idoso.

A reabilitação é um processo dinâmico, contínuo, progressivo e principalmente educativo, tendo como objetivos a restauração funcional do indivíduo, sua reintegração à família, a comunidade e a sociedade (RING, 1994).

Conforme salienta BRUMEL-SMITH (1997), o processo de reabilitação do idoso geralmente não é o mesmo quando comparado a uma pessoa jovem com o mesmo diagnóstico. As etapas são as mesmas, embora certos aspectos destas sejam diferentes quando trata-se de uma pessoa idosa. A autora, aponta os seguintes passos da reabilitação:
- estabilizar o problema primário e prevenir complicações secundárias - pode ser muitas vezes uma tarefa difícil com o idoso, frente a presença de múltiplas afecções; à medida que se envelhece, aparecem as doenças crônicas, caracterizadas principalmente pela hipertensão arterial, vasculopatias, artropatias, diabetes, entre outras, as quais podem desencadear limitações funcionais (RING, 1994); - restaurar a função perdida - embora a causa da perda de uma determinada função possa nunca ser resolvida, o idoso pode adquirir maior independência total ou parcial, compatível com seu estilo de vida; com o decorrer do processo de envelhecimento as funções corporais vão se alterando, podendo levar a um comprometimento da capacidade funcional, ou seja, do desempenho funcional para as atividades básicas e diárias (RING, 1994);

- promover adaptação da pessoa ao seu ambiente - os idosos muitas vezes apresentam dificuldade para aceitar a possibilidade de viver com uma incapacidade; nesta idade é comum ocorrer acentuação das características e crenças pessoais, étnicas, as quais influenciam os eventos da vida diária, inclusive a funcionalidade (RING, 1994); - adaptar o ambiente à pessoa - o processo de adaptação pode envolver alterações no ambiente nem sempre fáceis de serem realizadas frente a escassez de recursos financeiros e de apoio familiar;

- promover adaptação familiar - um dos principais sistemas de apoio ao idoso é a família, verificado por DIOGO (1990); CAVALCANTI (1991) e DIOGO et al. (1994), junto a pacientes com amputação. A reintegração de um jovem é muito diferente a do idoso, pois o jovem tem a sua frente uma função social mais claramente

\footnotetext{
* Enfermeira. Professor Assistente Doutor do Departamento de Enfermagem da Faculdade de Ciências Médicas da Universidade
} Estadual de Campinas. Membro do Grupo de Amputados da Unidade de Órteses e Próteses do Hospital das Clínicas - UNICAMP 
definida quando comparada ao idoso e de maior importância na sociedade. Embora a tendência atual seja de mudar este cenário, a reabilitação do idoso sofre a influência de vários preconceitos sociais, vinculados à fragilidade física, a presença de incapacidades funcionais, a incapacidade de aprendizagem e ao "peso" social decorrente da sua improdutividade (DIOGO \& CAMPEDELLI,1992; DIOGO,1997).

Portanto, pouco é investido na sua reabilitação, o que leva muitas vezes a uma "acomodação" por parte do idoso e a uma aceitação no sentido de resignação, por pensar que realmente já tenha cumprido seu papel social, não restando nada a fazer a não ser ajudar os membros da família na medida do possível e não atrapalhar a rotina diária. A família do idoso, principalmente daquele fragilizado e dependente, na maioria das vezes procura arranjos entre seus membros e possíveis adequações do contexto, visando o atendimento das suas necessidades. Buscam ainda alternativas de suporte social para o apoio no domicílio, numa tentativa de mantê-lo no seu ambiente, na sua comunidade, antes de pensar em institucionalizá10 .

Entretanto, nossa prática com idosos submetidos à amputação de membros inferiores, tem demonstrado que o idoso apresenta um grande potencial para a reabilitação, principalmente quando não existe outro comprometimento de ordem física ou mental. Assim, no caso de amputação por tumor, por exemplo, se o idoso estiver clinicamente bem e não apresenta contra indicação por parte do cardiologista, ele poderá desenvolver o programa de reabilitação até conseguir sua independência funcional utilizando uma prótese.

Outro ponto, salientado por BUTLER (1991), refere-se a hospitalização do idoso. A inatividade imposta durante este período, resulta no aumento da perda funcional, gerando um ciclo vicioso no qual o idoso tornase menos capaz de retornar ao seu nível de atividades anterior e com maior risco de adquirir problemas físicos adicionais. Neste sentido, acreditamos que a reabilitação é um processo contínuo, que deve ser iniciado a partir da hospitalização. A ênfase neste processo deve ser dada à capacidade remanescente do idoso, das suas habilidades, de maneira contínua, para ser adquirido o mais alto nível possível de independência e autocuidado.

Entre os princípios para a reabilitação geriátrica bem sucedida podemos destacar que é um processo no qual o cuidado é baseado na compreensão de que devem estar incorporados os parâmetros físico, emocional e social do idoso; a atuação da equipe multidisciplinar em relação a cada membro e interdisciplinar no processo; a ênfase na função a ser restaurada e nas funções remanescentes que necessitam ser modificadas e fortalecidas; é um processo fundamentalmente educativo.

A seguir abordaremos a questão da avaliação funcional do idoso que é um importante indicativo da qualidade da sua vida.

\section{AVALIAÇÃO FUNCIONAL DO IDOSO}

Em gerontologia, a avaliação funcional é de extrema importância na reabilitação, sendo o desempenho nas atividades da vida diária o parâmetro amplamente aceito e reconhecido.

A avaliação da capacidade funcional dos idosos permite a enfermeira e aos demais membros da equipe multidisciplinar uma visão mais precisa quanto à severidade da doença e o impacto da comorbidade. Assim, entende-se como avaliação funcional, a designação dada para uma função específica, a capacidade de se autocuidar e atender as necessidades básicas diárias ou seja, as "atividades da vida diária"(AVD). Entre as clássicas AVD propostas inicialmente por Sidney Katz incluem-se a capacidade para alimentar-se, transferir-se, vestir-se, banhar-se, ter continência e usar o banheiro, denominadas pelo autor e colaboradores como atividades básicas da vida diária (PASCHOAL, 1996). Outras atividades como preparar as refeições, lavar roupa, cuidar da casa, fazer compras, usar o telefone, usar o transporte, controlar o dinheiro e os medicamentos, são as "atividades instrumentais da vida diária"- AIVD, propostas por Lawton e Brody (PASCHOAL, 1996; PAULA et al., 1998).

A independência na realização das AVD é de grande importância na vida das pessoas pois envolve questões de natureza emocional, física e social. Independentemente da faixa etária, a dependência pode alterar a dinâmica familiar, os papéis desenvolvidos pelos seus membros, interferindo nas relações e no bem estar da pessoa dependente e dos seus familiares.

Neste contexto, a Organização Mundial de Saúde apresentou um modelo relacionado a capacidade funcional que traz conceitos e classifica os diferentes comprometimentos que os agravos à saúde podem trazer ao indivíduo, conforme é apontado por PAULA et al. (1998) e RING (1994): patologia - refere-se a doença, ao dano ou processo anormal que ocorre em um órgão; deficiência - toda perda ou anormalidade de estrutura ou função psicológica, fisiológica ou anatômica; incapacidade - é a dificuldade em realizar certas atividades da vida diária, como resultado de uma doença ou deficiência; desvantagem - é a conseqüência social da doença, da deficiência ou da incapacidade.

Estes conceitos se interligam a partir do pressuposto que a doença em um órgão, por exemplo, pode causar uma deficiência da sua função, que por sua vez pode levar a uma incapacidade funcional para com o seu meio ambiente e por último a uma desvantagem no 
contexto social como perda do emprego. No entanto, não há obrigatoriedade desta seqüência.

Para ilustrar estes conceitos, tomemos como exemplo um senhor de 75 anos de idade, com amputação suprapatelar de membro inferior esquerdo após um quadro agudo de trombose. Podemos dizer que a deficiência é a amputação de membro inferior esquerdo; a incapacidade relaciona-se a dificuldade de marcha, necessidade de ajuda para realizar as eliminações no banheiro, necessidade de ajuda para a alimentação (compra dos alimentos, preparo das refeições, acesso à cozinha); a desvantagem refere-se ao isolamento social, a necessidade de suporte familiar, a incapacidade de usar transporte coletivo, ao ambiente físico inadequado.

Conforme apontam PAULA et al. (1998), muitos testes e escalas de avaliação têm sido propostos para a avaliação funcional dos idosos, não existindo um modelo que atenda a todas as situações, pois segundo os autores a escolha de um método deve ser adequada e coerente a finalidade proposta.

\section{O PAPEL DA ENFERMEIRA NA REABILITAÇÃ̃O DO IDOSO}

A atuação da enfermeira junto ao idoso deve estar centrada na educação para a saúde, no "cuidar" tendo como base o conhecimento do processo de senescência e senilidade e no retorno da capacidade funcional para a realização das suas atividades, com objetivo de atender às suas necessidades básicas e alcançar sua independência e felicidade (CAMPEDELLI, 1983). Tal colocação embasa a assistência de enfermagem na situação de saúde e de doença, assim como direciona as ações de enfermagem num processo de reabilitação que vise o autocuidado. Neste processo, a enfermeira e demais profissionais envolvidos, devem atuar junto ao idoso e seus familiares, apoiando suas decisões, ajudando-os a aceitar as alterações na imagem corporal quando existentes, num processo educativo e congruente às necessidades individuais (DRENCH, 1994). O diagnóstico da doença do idoso pode não ter tanta importância quanto as conseqüências dele na qualidade de vida do idoso, ou seja, na sua capacidade funcional e na manutenção da independência para as atividades diárias.

A avaliação funcional do idoso faz parte do cuidado de enfermagem, com ênfase na pessoa e nos sistemas de apoio que ela pode contar. Assim consideramos que a enfermeira, inserida numa equipe mutidisciplinar, deve assistir ao idoso de maneira individualizada, levando em consideração as suas limitações físicas, psíquicas e ambientais.
A assistência sistematizada de enfermagem nos permite identificar os problemas dos idosos de maneira individualizada, planejar, executar e avaliar o atendimento a cada situação. Para tanto, direcionando a assistência para nível ambulatorial, a consulta de enfermagem é uma atividade que atende a estas questões aqui colocadas, por meio da qual a enfermeira assume a responsabilidade quanto a ação de enfermagem a ser determinada frente aos problemas detectados e estabelece a sua intervenção. Tendo em vista o enfoque nas AVD durante o processo de reabilitação, optamos por adequar à nossa realidade $\mathrm{e}$ a nossa experiência, o modelo para a consulta de enfermagem, que avalia as atividades da vida, modelo este criado pela enfermeira Nancy Roper e posteriormente elaborado em conjunto com Logan e Tierney (ROPER et al.,1981; 1985).

Um dos pressupostos deste modelo é que as pessoas que necessitam de enfermagem, têm alguns problemas de saúde, atual ou em potencial, os quais produzem uma ou mais alterações na vida diária ou seja, no desempenho das atividades da vida. O modelo proposto compreende as fases da metodologia assistencial propriamente dita, ou seja: Avaliação das atividades da vida, Planejamento da assistência de enfermagem, Intervenção de enfermagem e Avaliação da assistência de enfermagem prestada.

Assim sendo, durante a consulta de enfermagem, além do levantamento relativo aos dados pessoais, de saúde e da família, inclui-se a "Avaliação das atividades da vida". Cada atividade deve ser avaliada em termos da função e ação, buscando a identificação de rotinas anteriores, execução atual e problemas atuais e em potencial do idoso. Após a análise dos dados, os problemas são identificados e escritos.

Para a elaboração do "Planejamento da assistência de enfermagem", devemos considerar os recursos disponíveis em relação ao ambiente físico, aos equipamentos como o uso de acessórios e ao recurso pessoal, compreendido como enfermagem, família, serviços de apoio, entre outros. O plano de enfermagem não é estático e portanto, requer uma revisão contínua e coleta de dados adicionais a partir da avaliação inicial. Os objetivos a serem alcançados são traçados junto ao idoso, em termos do seu desempenho esperado após a intervenção de enfermagem.

$\mathrm{Na}$ fase de "Intervenção de enfermagem", a enfermeira seleciona e realiza as ações de enfermagem, tendo como objetivos a prevenção de problemas, a promoção de conforto físico e psíquico e a diminuição da dependência do idoso, tornando-o habilitado a procurar ajuda para atender sua responsabilidade para o autocuidado. As intervenções de enfermagem devem contudo, estar centradas nas rotinas do idoso, procurando minimizar os problemas relacionados às AVD. Estão 
incluídas ainda as ações relativas aos cuidados técnicos como curativos, medicações e outros procedimentos.

Para a última fase, identificada como "Avaliação da assistência de enfermagem prestada", o critério a ser utilizado é o desempenho do idoso em cada atividade frente ao alcance dos objetivos traçados previamente.

As AVD propostas para a consulta de enfermagem na reabilitação do idoso derivadas do trabalho por nós desenvolvido com idosos submetidos à amputação de membros inferiores são (DIOGO, 1993b): manutenção do ambiente seguro (características da habitação); locomoção, atividades físicas e esforços; alimentação e hidratação; eliminações; higiene corporal; ato de vestir-se; atividades realizadas em casa; sono e repouso; lazer e recreação; expressão da sexualidade; comunicação e uso de transportes.

Estas atividades devem ser avaliadas no sentido do desempenho do idoso e na funcionalidade de cada uma, com o objetivo de descobrir rotinas anteriores, $o$ que ele pode e não pode fazer por si próprio em relação a cada atividade e ainda quanto a alterações e problemas presentes.

Acrescida a esta avaliação faz-se necessário o exame físico sumário direcionado para a identificação de problemas que possam interferir no processo de reabilitação do idoso.

Com a finalidade de avaliar o nível de dependência na realização das AVD, entendida como a necessidade de ajuda de outra pessoa para o desempenho de cada atividade, consideramos importante destinar uma parte do modelo, sob a forma de quadro, para essa avaliação, visando facilitar a visualização do grau de dependência para cada uma delas. Este quadro contém lacunas referentes à data e o grau de dependência para cada AVD (independência, dependência ou dependência parcial) relacionados a avaliação realizada na parte anterior. Assim, em cada consulta de enfermagem com o idoso e seu familiar, conseguimos avaliar seu progresso ou não no desempenho de cada atividade.

Após a avaliação inicial, esta parte é retomada nos contatos subseqüentes com o objetivo de identificar as alterações ocorridas no grau de dependência do idoso, permitindo assim uma avaliação contínua para cada atividade.

A última parte da consulta de enfermagem corresponde ao Plano assistencial de enfermagem, compreendendo as fases de Planejamento da assistência e Intervenção de enfermagem. Neste momento a enfermeira identifica os problemas e registra as ações de enfermagem que se fazem necessárias, possibilitando deste modo dar prosseguimento a sua assistência. No retorno do idoso é dada maior ênfase ao Plano assistencial de enfermagem no qual é realizada a avaliação das ações de enfermagem realizadas e ao quadro de Avaliação da dependência na realização das AVD. As demais partes do instrumento são abordadas de maneira sucinta, avaliando a presença de novos problemas e outras intervenções de enfermagem que se façam necessárias.

\section{FASES DA REABILITACÃO GERIÁTRICA E A ATUAÇÃO DA ENFERMEIRA}

Segundo TUCKER (1993), as enfermeiras desenvolvem seu plano de assistência com base em quatro estágios da reabilitação do idoso: ajustamento, progresso, platô e alta.

No estágio de ajustamento inclui-se o processo de admissão, orientações quanto a sua reabilitação e quanto ao protocolo a ser seguido. O objetivo nesta fase é discutir as expectativas do idoso e a elaboração do plano. A avaliação é realizada também pelos demais membros da equipe, tendo-se o cuidado de não "estressar" o idoso com sobrecarga de informações. Nesta etapa é importante avaliar a motivação do idoso para participar do processo, assim como o suporte e envolvimento de familiares e amigos, os quais são fatores fundamentais para o sucesso do programa. Por meio da consulta de enfermagem a enfermeira avalia estas questões, assim como avalia a realização das AVD e o grau de dependência para cada uma delas e ainda com base nos problemas identificados, planeja e executa as ações de enfermagem pertinentes a cada idoso. Cabe ressaltar a importância de se dar ênfase na capacidade remanescente do idoso e ajudá-lo a se ajustar à nova situação.

A fase de progresso é caracterizada pela familiarização do idoso com a equipe e com o programa participando das sessões terapêuticas. É uma etapa dinâmica, altamente gratificante para todos os membros da equipe, para o idoso e familiares envolvidos. A enfermeira participa ativamente avaliando as respostas do idoso, realizando ações de enfermagem, promovendo suporte psicossocial e atuando principalmente no processo educativo.

A fase seguinte, ou seja, o platô, ocorre quando o progresso diminui, embora as mudanças continuem a acontecer. Pode ser uma longa etapa no processo de reabilitação, na qual o idoso se capacita para realizar as funções, com segurança em sua moradia. Neste momento, a participação da enfermeira está direcionada as ações anteriormente descritas, além de estimular o envolvimento do idoso, prevenindo desânimo. Para tanto são necessárias habilidades na comunicação para manter a motivação do idoso e o seu envolvimento e da família no processo.

A última fase, a alta, é um estágio altamente variado, em função da individualidade do idoso e da 
dificuldade em se determinar com precisão o momento da alta, ou seja, em qual ponto e data ele estará "pronto". Outrossim, o senso de protecionismo da equipe tende a prevalecer. A avaliação domiciliar e dos recursos da comunidade deve ser realizada, assim como a formação de grupos de orientação para a família e cuidadores, com objetivo de prepará-los para a alta e ainda promover troca de experiências. Nem sempre a orientação adequada coincide com a realidade do idoso em seu ambiente domiciliar e comunitário, sendo necessários arranjos e adaptações.

\section{PROBLEMAS FREQÜENTES NA REABILITAÇÃO DO IDOSO E A ATUAÇÃO DA ENFERMEIRA}

Conforme mencionamos anteriormente, o idoso pode apresentar vários problemas que levam ao comprometimento da sua reabilitação. TUCKER (1993) chama atenção a alguns deles ainda não apontados:

- frente a presença de múltiplas afecções, os idosos fazem uso geralmente de polifármacos, portanto, a enfermeira deve estar preparada para intervir, por meio de uma avaliação minuciosa, visando favorecer o progresso do idoso na reabilitação e seu estado de saúde; a adesão do idoso ao programa de reabilitação pode ser outro problema que requer da enfermeira principalmente habilidade na comunicação;

- dificuldades de transporte para o serviço de saúde ou ainda de acompanhante, tendo em vista a vida atribulada que as pessoas vivenciam hoje em dia;

- frustração relacionada à reabilitação, ou seja, o idoso pode apresentar uma expectativa muito alta não condizente com o programa. Temos observado por meio da nossa prática com idosos submetidos à amputação de membros inferiores que a expectativa que apresentam em colocar uma prótese está vinculada à idéia de que com o uso dela, a maioria dos problemas vivenciados serão resolvidos, sejam eles de natureza emocional, social ou de dependência para as AVD (DIOGO, 1993a,b). No entanto, se eventualmente, na avaliação dos profissionais não for indicada seu uso, seja por razões clínicas ou pela presença de outras incapacidades, o idoso freqüentemente abandona o programa, apresentando intensa frustração; - desânimo, depressão e idéia de suicídio, principalmente na fase de platô, quando diminui ou estaciona seu progresso, podendo ficar claro para ele que a alta do programa dificilmente será conseguida. Muitas vezes observamos durante a consulta de enfermagem, reações do idoso como crises de choro e desânimo, desejo de abandonar o programa, ou ainda, percepções próprias de não conseguir alta ou não atingir o progresso esperado.
Nessas situações é de extrema importância a enfermeira ser sensível para detectar estes problemas para interagir com o idoso, família e demais membros da equipe;

- sistemas de suporte - os familiares também apresentam dificuldades em aceitar as mudanças no idoso, a realidade diária (principalmente vinculadas a dependência do idoso) e as alterações dos papéis no contexto familiar. Isto nos leva a destacar a importância de se investigar as reações dos familiares e principalmente das pessoas mais envolvidas, ou seja, os cuidadores, que em muitas situações, encontram na enfermeira, a possibilidade de expressar seus sentimentos e dificuldades presentes no cuidado ao idoso conforme ressalta WEEKS (1995), para também poder atuar com essas pessoas;

- necessidade de educação à saúde - alguns idosos desenvolvem ao longo da sua vida, hábitos os quais não são facilmente alterados e portanto, conseguir as mudanças necessárias pode ser um desafio. Além disso, outros problemas podem estar presentes como fatores de risco para acidentes, uso inadequado de medicações, desconhecimento do processo de senescência e mitos relacionados à aprendizagem do idoso. Assim estas áreas demandam orientação e "negociação" por parte da enfermeira, que no seu papel de educadora necessita utilizar de habilidades para o ensino e formar verdadeiras "sociedades" com os cuidadores;

- relutância do idoso à alta - o medo de se desvincular do programa é um problema do idoso a qual a enfermeira pode ajudá-lo a superar. Esse medo pode estar relacionado à expectativa de solidão após longo período de convivência com os membros da equipe, ao enfrentamento solitário do cotidiano, além do companheirismo vivenciado, no local da reabilitação, com outros pacientes. Portanto, estas manifestações requerem uma abordagem tanto da enfermeira quanto dos demais membros da equipe, demonstrando apoio e disponibilidade para ouvir seus sentimentos e suas dificuldades. A manutenção de um vínculo, seja ele esporádico, pode favorecer segurança para o idoso, o qual pode participar em grupos educativos relatando sua experiência e assim estimulando-o a se sentir útil e importante no processo de reabilitação de outros idosos.

\section{CONSIDERAÇÕES FINAIS}

A reabilitação geriátrica é uma das faces da Enfermagem geriátrica e gerontológica. A atuação de enfermeira na equipe multidisciplinar está centrada no processo educativo com o idoso e seus familiares, tendo como finalidade a sua independência funcional, a prevenção de complicações secundárias, sua adaptação e da família à nova situação. 
Por meio da assistência de enfermagem sistematizada, a enfermeira elabora, executa e avalia o Plano assistencial de enfermagem individualizado, respeitando os diferentes estágios da reabilitação em que o idoso pode se encontrar. No entanto é fundamental seu conhecimento sobre o processo de senescência e senilidade, sobre o contexto familiar e social do idoso, respeitando suas limitações e enfatizando seu potencial remanescente e sua capacidade para o autocuidado.
Lembrar ainda que cada idoso possui a sua história de vida, diferente de qualquer outra e o que pode significar qualidade de vida para ele pode ser diferente do que pode significar para o profissional da saúde. Assim é importante termos cuidado para não pensarmos e agirmos apenas sobre nossos próprios valores quando assistimos ao idoso (ou ainda outras pessoas). É uma área que requer ainda grande investimento no ensino, na pesquisa e na assistência.

\section{THE NURSE'S FUNCTION IN THE ELDERLY'S REHABILITATION}

As the elderly people in our country are increasing it becomes more and more frequent the elderly's presence in the services of health and especially of rehabilitation. We bring in this descriptive text the main aspects involved in the elderly's rehabilitation: the referring peculiarities to the rehabilitation in this age group, the functional evaluation as an important indicative of the life quality, the phases of the elderly's rehabilitation and the systematized nursing care through the nurse, inserted in the multidisciplinar team acts with the elderly patient and his family, in an individualized way.

KEY WORDS: geriatric rehabilitation, geriatric and gerontological nursing

\section{EL PAPEL DE LA ENFERMERA EN LA REHABILITACIÓN DEL ANCIANO}

Con el aumento creciente de los ancianos en nuestro país, se torna cada vez más frecuente la presencia del anciano en los servicios de salud y sobre todo de rehabilitación. Traemos en este texto descriptivo, los principales aspectos involucrados en la rehabilitación del anciano: las peculiaridades referentes a la rehabilitación en este grupo de edad, la evaluación funcional como un importante indicativo de la calidad de vida, las fases de la rehabilitación del anciano y de la atención sistematizada de enfermería por medio de la cual la enfermera, insertada en el equipo multidisciplinario actúa con el anciano y su familia, de manera individualizada.

TÉRMINOS CLAVES: rehabilitación geriátrica, enfermería geriátrica y gerontológica

\section{REFERÊNCIAS BIBLIOGRÁFICAS}

01. BRUMMEL-SMITH, K. Rehabilitation. In: HAM, R.J.; SLOANE, P.D. Primary care geriatrics case - based approach. 3ed. New York: Mosby, 1997. Cap. 11, p. 139-152.

02. BUTLER, M. Geriatric rehabilitation nursing. Rehabil.Nurs., v. 16, n. 6, p. 318-21, 1991.

03. CAMPEDELLI, M.C. Atuação da enfermagem em geriatria e gerontologia. Rev. Paul. Hosp., v. 31, n. 9/10, p. 198-200, 1983.

04. CAVALCANTI, M.C.T. Conversando com a pessoa a ser amputada: uma contribuição à psicologia médica. Campinas, 1991.276p. Tese (doutorado) - Faculdade de Ciências Médicas, Universidade Estadual de Campinas.

05. DIOGO, M.J.D’E. A problemática do idoso submetido à amputação de membros inferiores. São Paulo, 1990. 137p. Dissertação (Mestrado) - Escola de Enfermagem, Universidade de São Paulo.
06. DIOGO, M.J.D’E. Sentimentos relacionados com a auto-imagem de idosos submetidos à amputação de membros inferiores. Rev. Esc. Enfermagem USP, v. 27, n. 2, p. 296-308, 1993a.

07. DIOGO, M.J.D'E. A consulta de enfermagem centrada nas atividades da vida: proposta de assistência ao idoso com amputação de membros inferiores. São Paulo, 1993b. 167p. Tese (Doutorado) - Escola de Enfermagem, Universidade de São Paulo.

08. Diogo, M.J.D’E. A dinâmica dependênciaautonomia: possibilidades em idosos submetidos à amputação de membros inferiores. Rev.latinoam.enfermagem, v. 5, n. 1, p. 59-64, 1997.

09. DIOGO, M.J.D’E.; CAMPEDELLI, M.C. O idoso submetido à amputação de membros inferiores e as alterações nas atividades da vida diária. Rev.

Paul. Enfermagem, v. 11, n. 2, p. 92-9, 1992. 
10. DIOGO, M.J.D’E.; CAMPEDELLI, M.C.; CEOLIM, M.F. Alterações das atividades pessoais no idoso com amputação. Gerontologia, v. 2, n. 2, p. 5968, 1994.

11. DRENCH, M.E. Changes in body image secondary to disease and injury. Rehabil.Nurs., v. 19, n. 1, p. 31-6, 1994.

12. KALACHE, A. et al. O envelhecimento da população mundial: um desafio novo. Rev. Saúde Pública, v. 21, n. 3, p. 200-10, 1987.

13. PASCHOAL, S.M.P. Autonomia e independência. In: PAPALÉO NETTO, M. Gerontologia. São Paulo: Atheneu, 1996. Cap. 28, p. 313-23.

14. PAULA, J.A.M.; TAVARES, M. da C.G.C.F; DIOGO, M.J.D’E. Avaliação funcional em gerontologia. Gerontologia, v. 6, n. 2, p. 81-8, 1998.
15. RING, H. La rehabilitación en los ancianos. In.: ORGANIZACIÓN PANAMERICANA DE LA SALUD. La atención de los ancianos: un desafio para los años noventa. Washington: Elias Anzola Pérez, 1994. p. 279-88.(Publicación Científica, 546).

16. ROPER, N. et al. Learning to use the process of nursing. London: Churchill Livingstone, 1981. $119 \mathrm{p}$.

17. The elements of nursing. 2. ed. London: Churchill Livingstone, 1985. 378p.

18. TUCKER, N.J. Geriatric rehabilitation: nursing challange of the ' 90 s. Rehabil.Nurs., v. 18, n. 2, p. 114-6, 1993.

19. WEEKS, S.K. What are the educational needs of prospective family caregivers of newly disabled adults?. Rehabil.Nurs., v. 20, n. 5, p. 256-60, 1995. 